\title{
Predominance of Abdominal Visceral Adipose Tissue Reflects the Presence of Aortic Valve Calcification
}

\author{
Masayoshi Oikawa, Takashi Owada, Hiroyuki Yamauchi, Tomofumi Misaka, \\ Hirofumi Machii, Takayoshi Yamaki, Koichi Sugimoto, Hiroyuki Kunii, \\ Kazuhiko Nakazato, Hitoshi Suzuki, Shu-ichi Saitoh, and Yasuchika Takeishi \\ Department of Cardiology and Hematology, Fukushima Medical University, 1 Hikarigaoka, Fukushima 960-1295, Japan \\ Correspondence should be addressed to Masayoshi Oikawa; moikawa@fmu.ac.jp
}

Received 6 August 2015; Revised 2 December 2015; Accepted 28 December 2015

Academic Editor: Ramazan Akdemir

Copyright (C) 2016 Masayoshi Oikawa et al. This is an open access article distributed under the Creative Commons Attribution License, which permits unrestricted use, distribution, and reproduction in any medium, provided the original work is properly cited.

Background. Aortic valve calcification (AVC) is a common feature of aging and is related to coronary artery disease. Although abdominal visceral adipose tissue (VAT) plays fundamental roles in coronary artery disease, the relationship between abdominal VAT and AVC is not fully understood. Methods. We investigated 259 patients who underwent cardiac and abdominal computed tomography (CT). AVC was defined as calcified lesion on the aortic valve by CT. \%abdominal VAT was calculated as abdominal VAT area/total adipose tissue area. Results. AVC was detected in 75 patients, and these patients showed higher \%abdominal VAT (44\% versus $38 \%, p<0.05)$ compared to those without AVC. When the cutoff value of \%abdominal VAT was set at $40.9 \%$, the area under the curve to diagnose AVC was 0.626. Multivariable logistic regression analysis showed that age (OR 1.120, 95\% CI 1.078-1.168, $p<0.01$ ), diabetes (OR 2.587, 95\% CI 1.323-5.130, $p<0.01$ ), and \%abdominal VAT (OR 1.032, 95\% CI 1.003-1.065, $p<0.05$ ) were independent risk factors for AVC. The net reclassification improvement value for detecting AVC was increased when \%abdominal VAT was added to the model: 0.5093 (95\% CI 0.2489-0.7697, $p<0.01$ ). Conclusion. We determined that predominance of VAT is associated with AVC.

\section{Introduction}

Aortic valve calcification (AVC) is a common feature of aging and is considered as atherosclerotic changes of the aortic valve [1]. Because early-stage AVC does not present any symptoms, many cases are incidentally diagnosed by cardiac imaging examination for other cardiac or pulmonary diseases. Although the rate of progression of AVC to clinical aortic stenosis is low, AVC is independently associated with cardiovascular mortality $[2,3]$. Traditional cardiovascular risk factors are associated with the development of AVC, and obesity is also related to its progression [1]. Central obesity is an indispensable factor for the diagnosis of metabolic syndrome, which is related to the progression of not only coronary artery disease but also AVC [4]. Abdominal visceral adipose tissue (VAT) produces various inflammatory cytokines that cause the development of insulin resistance, which is a fundamental characteristic of metabolic syndrome
[5]. Although VAT has been reported to be associated with coronary artery calcification and abdominal aorta calcification [6, 7], few reports have focused on AVC. The purpose of this study was to evaluate the importance of abdominal VAT in the development of AVC.

\section{Materials and Methods}

2.1. Subjects. We enrolled 259 patients who had undergone 64-slice computed tomography (CT) angiography for the diagnosis of coronary artery disease from January 2013 through December 2014. Hypertension was defined as the recent use of antihypertensive drugs, systolic blood pressure $\geq 140 \mathrm{mmHg}$, and/or diastolic pressure $\geq 90 \mathrm{mmHg}$. Diabetes was defined as the recent use of insulin or antidiabetic drugs, fasting blood glucose $\geq 126 \mathrm{mg} / \mathrm{dL}$, and/or hemoglobin $A_{1 c} \geq 6.5 \%$. Dyslipidemia was defined as the recent use of cholesterol-lowering drugs, triglyceride $\geq 150 \mathrm{mg} / \mathrm{dL}$, 
TABLE 1: Patient characteristics.

\begin{tabular}{lcc}
\hline & Non-AVC $(n=184)$ & AVC $(n=75)$ \\
\hline Age $($ years $)$ & $64 \pm 12$ & $75 \pm 8^{* *}$ \\
BMI $\left(\mathrm{kg} / \mathrm{m}^{2}\right)$ & $24.6 \pm 3.9$ & $23.7 \pm 4.4$ \\
Male $(n, \%)$ & $113,61 \%$ & $44,59 \%$ \\
Hypertension $(n, \%)$ & $106,58 \%$ & $53,71 \%$ \\
Dyslipidemia $(n, \%)$ & $96,52 \%$ & $36,48 \%$ \\
Diabetes $(n, \%)$ & $41,22 \%$ & $31,41 \%{ }^{* *}$ \\
Smoking history $(n, \%)$ & $72,39 \%$ & $32,43 \%$ \\
Abdominal VAT area $\left(\mathrm{cm}^{2}\right)$ & $89.7[67.8-127.0]$ & $94.1[50.0-138.7]$ \\
Subcutaneous adipose tissue area $\left(\mathrm{cm}^{2}\right)$ & $110.0[24.7-151.1]$ & $80.8[7.4-115.0]^{* *}$ \\
Total adipose tissue area $\left(\mathrm{cm}^{2}\right)$ & $191.2[45.2-254.9]$ & $140.0[18.3-204.4]^{* *}$ \\
Coronary arteriosclerosis $(n, \%)$ & $111,60 \%$ & $66,88 \%{ }^{* *}$ \\
\hline
\end{tabular}

Values are expressed as median with interquartile ranges.

AVC, aortic valve calcification; BMI, body mass index; VAT, visceral adipose tissue; ${ }^{* *} p<0.01$ versus non-AVC group.

low-density lipoprotein cholesterol $\geq 140 \mathrm{mg} / \mathrm{dL}$, and/or high-density lipoprotein cholesterol $\leq 40 \mathrm{mg} / \mathrm{dL}$. Patients who had poor image data or a history of open heart surgery or acute coronary syndrome were excluded. The study protocol was approved by the institutional ethics committee, and informed consent was obtained from all study subjects.

2.2. CT Scan Protocol. CT examinations were performed using a 64-slice CT scanner (Aquilion 64, Toshiba Medical Systems Co., Ltd., Tochigi, Japan) with a collimation of $64 \times$ $0.5 \mathrm{~mm}$, pixel size of $0.39 \times 0.39 \mathrm{~mm}$, gantry rotation time of $350 \mathrm{msec}$, and tube voltage of $120 \mathrm{kV}$. The protocol of premedication and CT scan have been described previously [8]. A noncontrast scan was performed at the level of the umbilicus in order to assess the abdominal VAT area. Only one slice at the umbilical level was captured for the sake of reducing radiation exposure. The reconstructed image data were analyzed by a computer workstation (Ziostation2, Ziosoft Inc., Tokyo, Japan).

2.3. Evaluation of CT Findings. The areas of abdominal VAT and subcutaneous adipose tissue were measured by an application software (Fat Measurement, Toshiba Medical Systems Co., Ltd., Tochigi, Japan) as previously described [8]. \%abdominal VAT area was calculated as the ratio of abdominal VAT area to the total adipose tissue area and multiplied by 100. Epicardial adipose tissue (EAT) area was measured at the level of four-chamber view of the heart. EAT was defined as any tissues presenting between -230 Hounsfield unit (HU) and $-30 \mathrm{HU}$ enclosed by the visceral pericardium [8]. Coronary arteriosclerosis was determined as any visible coronary calcification or atheromatous plaque on a coronary artery. We defined AVC as any visible structures presenting more than $130 \mathrm{HU}$ detected on the aortic valve.

2.4. Statistics. Data are expressed as mean with standard deviation. When the data were not normally distributed, median with interquartile ranges was reported. Student's $t$-test was used for normally distributed data, and the MannWhitney test was used for unequally distributed data. Logistic regression analysis was performed on anthropometric and clinical variables to identify correlates of AVC. The variables of $p<0.10$ in the univariable logistic analysis were analyzed by the multivariable analysis. The improvement by adding \%abdominal VAT to discrimination and net reclassification of risks was assessed by comparing the area under the curve (AUC) of receiver operator characteristics and the estimation of both integrated discrimination improvement (IDI) and net reclassification improvement (NRI) [9]. All tests were two-tailed and $p$ values $<0.05$ were considered statistically significant. All statistical analyses were performed using $\mathrm{R}$ software packages version 3.1.2 (R Core Team 2014, Vienna, Austria).

\section{Results}

Patient characteristics are displayed in Table 1. Out of 259 patients, 75 were found to have AVC. Compared to the patients with non-AVC, those with AVC were older and had a higher prevalence of diabetes. Consistent with previous reports, the prevalence of coronary arteriosclerosis was higher in the AVC group. Although the size of abdominal VAT areas was similar between the AVC and non-AVC groups (Table 1), the \%abdominal VAT area was larger in the AVC group (Figure 1(a)), suggesting the predominance of abdominal visceral fat in the AVC group.

Figure 1(b) shows a receiver operator characteristic curve for the detection of AVC using a variable of \%abdominal VAT. When the cutoff value was determined as $40.9 \%$, diagnostic accuracy was as follows: sensitivity 0.653 , specificity 0.625 , positive predictive value 0.184 , negative predictive value 0.585, and AUC 0.626.

Because EAT was also categorized as ectopic visceral adipose tissue and was related to the development of coronary arteriosclerosis $[8,10]$, we next measured EAT area. As expected, there was a positive correlation between EAT area and abdominal VAT area (Figure 2(a)). Unlike abdominal 


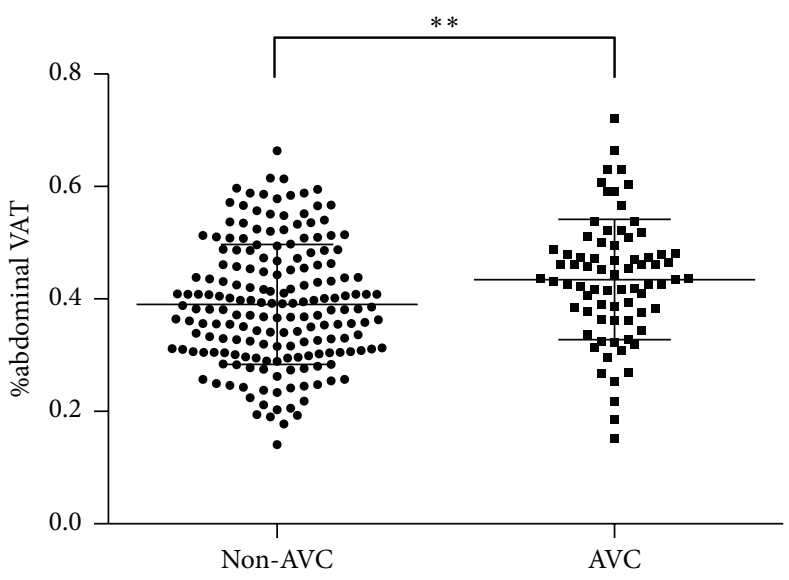

(a)

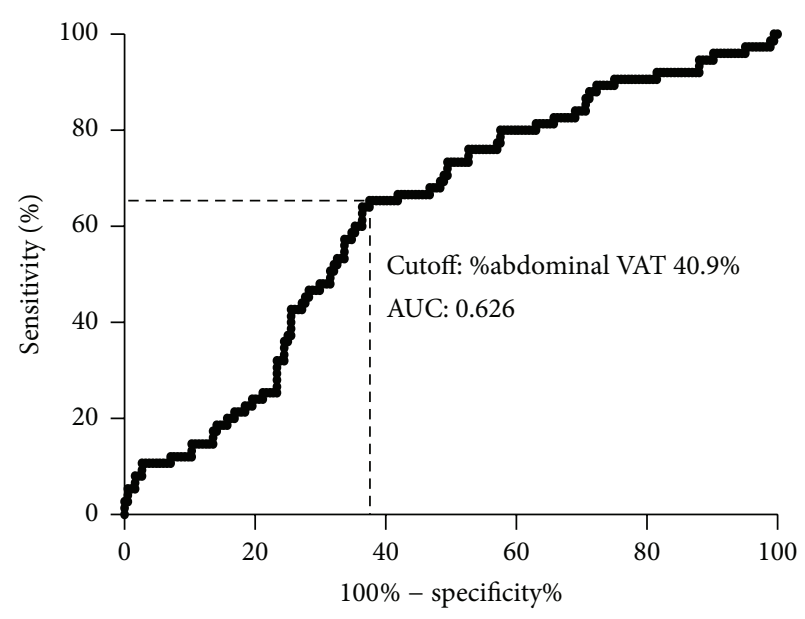

(b)

Figure 1: Relationship between \%abdominal VAT and AVC. (a) Difference of \%abdominal VAT between the non-AVC and AVC groups. Each point represents \%abdominal VAT area of the patients. Error bars; mean with standard deviation. ${ }^{* *} p<0.01$ versus non-AVC. (b) ROC curve analysis for the detection of AVC. AUC was calculated when the cutoff value of \%abdominal VAT was set at $40.9 \%$.

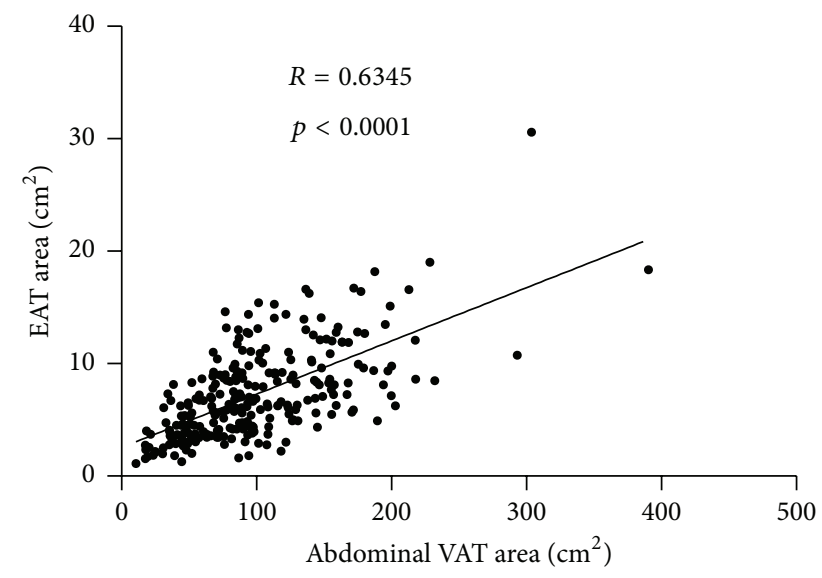

(a)

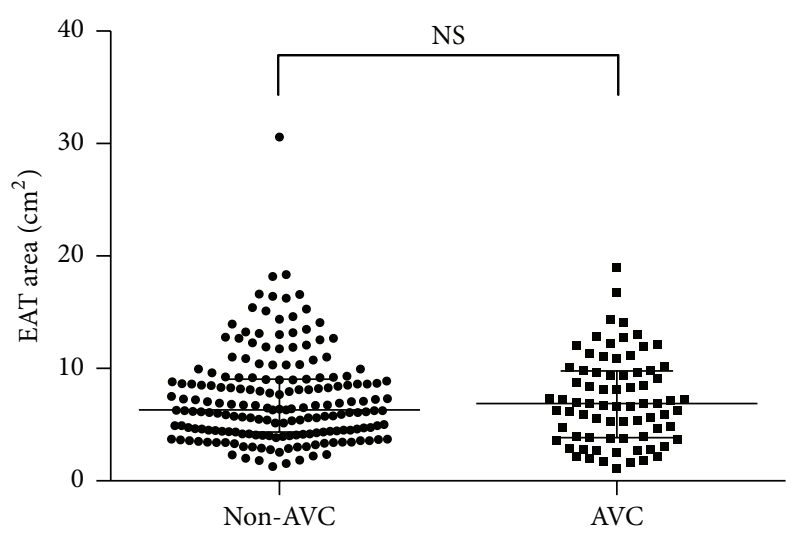

(b)

FIGURE 2: Relationship between EAT and AVC. (a) Correlation between abdominal VAT and EAT. Each point represents abdominal VAT and EAT area of the patients. (b) Difference of EAT area between the non-AVC and AVC groups. Each point represents EAT area of the patients. Error bars; median with interquartile ranges.

VAT, there was no difference in EAT area between the presence and absence of AVC (Figure 2(b)).

As shown in Table 2, univariable logistic regression analysis revealed that age, diabetes, and \%abdominal VAT were associated with AVC. Multivariable logistic regression analysis indicated that age, diabetes, and \%abdominal VAT were independent variables for predicting AVC.

Although it did not reach statistical significance, value of AUC was increased by $1.1 \%(p=0.25)$ when $\%$ abdominal VAT was added to the model. To assess the importance of adding \%abdominal VAT more precisely, NRI and IDI were calculated using the variables with or without \%abdominal VAT. The values of NRI for detecting AVC reached statistical significance when \%abdominal VAT was added to the model: 0.5093 (95\% CI 0.2489-0.7697, $p<0.001$ ) for NRI and 0.0116 (95\% CI $-0.0016-0.0347, p=0.07)$ for IDI.

\section{Discussion}

In the present study, we demonstrated that increased \%abdominal VAT is associated with the presence of AVC. In addition, \%abdominal VAT was an independent risk factor of the presence of AVC, and the value of NRI and IDI supported the significance of \%abdominal VAT in the development of AVC.

4.1. Association of Abdominal VAT and AVC. Several reports showed that oxidative stress, arising from an increased production of free radicals and decreased antioxidant system, is an initial causative stimulus for AVC [1]. Superoxide dismutase (SOD) activity and expression of SOD isoforms were reduced in calcified lesions of human aortic valves [11]. Superoxide was present more abundantly in stenosed 
TABLE 2: Association with the presence of aortic valve calcification.

\begin{tabular}{|c|c|c|c|c|}
\hline & \multicolumn{2}{|c|}{ Univariate } & \multicolumn{2}{|c|}{ Multivariate } \\
\hline & OR $(95 \% \mathrm{CI})$ & $p$ value & OR $(95 \% \mathrm{CI})$ & $p$ value \\
\hline Age, per 1-year increase & $1.119(1.081-1.164)$ & $<0.001$ & $1.120(1.078-1.168)$ & $<0.001$ \\
\hline BMI, per $1 \mathrm{~kg} / \mathrm{m}^{2}$ increase & $0.939(0.872-1.007)$ & 0.085 & $0.968(0.889-1.050)$ & 0.446 \\
\hline Male & $0.892(0.517-1.549)$ & 0.681 & & \\
\hline Hypertension & $1.772(1.006-3.201)$ & 0.052 & $1.124(0.580-2.200)$ & 0.730 \\
\hline Dyslipidemia & $0.846(0.493-1.448)$ & 0.542 & & \\
\hline Diabetes & $2.457(1.379-4.379)$ & 0.002 & $2.587(1.323-5.130)$ & 0.006 \\
\hline Smoking history & $1.158(0.669-1.994)$ & 0.599 & & \\
\hline \%abdominal VAT, per $1 \%$ increase & $1.039(1.013-1.067)$ & 0.003 & $1.032(1.003-1.065)$ & 0.033 \\
\hline
\end{tabular}

OR, odds ratio; CI, confidence interval; BMI, body mass index; VAT, visceral adipose tissue.

aortic valves of hypercholesterolemic mouse hearts [12]. Furthermore, not only reactive oxygen species (ROS) but also inflammatory cytokines are associated with AVC. In patients with aortic valve sclerosis, interleukin- $1 \beta$ was upregulated in leukocytes that had infiltrated to the calcified aortic valve [13]. There is a growing body of evidence showing that adipose tissues are related to the production of both oxidative stress and inflammatory cytokines. In obese mice model, ROS were increased with augmented expression of nicotinamide adenine dinucleotide phosphate (NADPH) oxidase, tumor necrosis factor- $\alpha$, and plasminogen activator inhibitor-1 in accumulated adipose tissues [14]. Similarly, nondiabetic obese human subjects showed increased ROS production accompanied with an increase in NADPH oxidase activity [14]. Although we did not have the data regarding the oxidative stress and inflammatory cytokines in this study, these results indicate that increased adipose tissues may contribute to the development of AVC by inducing oxidative stress and inflammation.

In the present study, however, a simple measurement of the abdominal VAT area was similar between the AVC and non-AVC groups. This apparent inconsistency may be explained by the predominance of abdominal VAT. The amount of VAT tends to increase in correlation with total adipose tissue volume, but the proportion of subcutaneous adipose tissue (SAT) and VAT differs from person to person. When energy intake surpasses calorie expenditure, SAT stores excess energy by recruiting new precursor cells, leading to hyperplastic obesity [15]. This type of subcutaneous obesity is characterized by low ectopic fat volume and a normal metabolic profile [16]. However, once recruitment ability of the precursor cells is impaired, adipocyte hypertrophy occurs, and excess energy is stored as ectopic fat [16]. Increased ectopic fat depot, typically abdominal VAT, is strongly related to insulin resistance, producing inflammatory cytokines, and the development of type II diabetes mellitus [16]. Because \%abdominal VAT area reflected both SAT and VAT, it may be more feasible parameter than simple assessment of abdominal VAT area. Consistent with this concept, the multivariable logistic analysis showed \%abdominal VAT was an independent risk factor of AVC. Moreover, the value of NRI reached statistical significance, indicating the close relationship between \%abdominal VAT and AVC. Not only abdominal VAT but also EAT is associated with arteriosclerosis. In the present study, there was no difference in EAT area between the presence and absence of AVC. The mechanisms by which EAT promotes coronary artery disease are partly explained by a direct exposure of inflammatory cytokines produced by EAT to coronary vessel wall $[17,18]$. From its viewpoint, aortic valve is apart from EAT, and the volume of EAT is much less compared to abdominal visceral fat. Therefore, we speculate that EAT showed no relationship with presence of AVC. Given that AVC is associated with cardiovascular mortality in spite of hemodynamic insignificance, the presence of AVC may exhibit an early sign of metabolic disturbance affecting the cardiovascular system. Thus, we should take into consideration a detailed assessment of the patient's metabolic profile as well as proper medical management in cases where AVC is detected.

4.2. Study Limitations. First, our results are based on a relatively small number of patients and were obtained after excluding lesions with inadequate image quality from analysis. Second, the patients included in the study might have possessed different clinical backgrounds from normal subjects, as they have symptoms or clinical features of suspected coronary artery disease. This raises the possibility that they were likely to be more exposed to arteriosclerotic risk factors than the normal population. Third, Agatston score is useful to classify the severity of aortic valve calcification, but we could not calculate the score because nonenhanced CT was not performed before coronary CT angiography. The relationship between the amount of VAT and severity of AVC remains to be elucidated in the future study. Fourth, the growing body of evidence suggested that both oxidative stress and inflammatory cytokines play important roles in initiating aortic valve calcification. However, unfortunately, the present study was not able to exhibit the direct evidence of the induction of oxidative stress and production of inflammatory cytokines because no data for C-reactive protein, sedimentation rate, myeloperoxidase, and so forth were available. This point should be further examined in the future study. 


\section{Conclusions}

The present study revealed the relationship between the presence of AVC and abdominal visceral adiposity. Given the importance of AVC for cardiovascular mortality, we should consider precise assessment of metabolic profile and medical management when AVC is detected.

\section{Abbreviations}

AVC: Aortic valve calcification

VAT: Visceral adipose tissue

CT: $\quad$ Computed tomography

EAT: Epicardial adipose tissue

HU: Hounsfield unit

AUC: Area under the curve

IDI: Integrated discrimination improvement

NRI: Net reclassification improvement

SOD: $\quad$ Superoxide dismutase

ROS: $\quad$ Reactive oxygen species

NADPH: Nicotinamide adenine dinucleotide phosphate

SAT: $\quad$ Subcutaneous adipose tissue.

\section{Conflict of Interests}

The authors declare that there is no conflict of interests regarding the publication of this paper.

\section{Acknowledgment}

The authors acknowledge Professor Tetsuya Ohira (Department of Epidemiology, Radiation Medical Center for the Fukushima Health Management Survey, Fukushima Medical University) for his advice on medical statistics.

\section{References}

[1] N. M. Rajamannan, F. J. Evans, E. Aikawa et al., "Calcific aortic valve disease: not simply a degenerative process a review and agenda for research from the national heart and lung and blood institute aortic stenosis working group executive summary: calcific aortic valve disease-2011 update," Circulation, vol. 124, no. 16, pp. 1783-1791, 2011.

[2] S. Coffey, B. Cox, and M. J. A. Williams, "The prevalence, incidence, progression, and risks of aortic valve sclerosis: a systematic review and meta-analysis," Journal of the American College of Cardiology, vol. 63, no. 25, pp. 2852-2861, 2014.

[3] C. M. Otto, B. K. Lind, D. W. Kitzman, B. J. Gersh, and D. S. Siscovick, "Association of aortic-valve sclerosis with cardiovascular mortality and morbidity in the elderly" The New England Journal of Medicine, vol. 341, no. 3, pp. 142-147, 1999.

[4] R. Katz, M. J. Budoff, J. Takasu et al., "Relationship of metabolic syndrome with incident aortic valve calcium and aortic valve calcium progression: the Multi-Ethnic Study of Atherosclerosis (MESA)," Diabetes, vol. 58, no. 4, pp. 813-819, 2009.

[5] J.-P. Després, I. Lemieux, J. Bergeron et al., "Abdominal obesity and the metabolic syndrome: contribution to global cardiometabolic risk," Arteriosclerosis, Thrombosis, and Vascular Biology, vol. 28, no. 6, pp. 1039-1049, 2008.
[6] C. S. Fox, S.-J. Hwang, J. M. Massaro et al., "Relation of subcutaneous and visceral adipose tissue to coronary and abdominal aortic calcium (from the Framingham Heart study)," The American Journal of Cardiology, vol. 104, no. 4, pp. 543-547, 2009.

[7] J. Golledge, R. Jayalath, L. Oliver, A. Parr, L. Schurgers, and P. Clancy, "Relationship between CT anthropometric measurements, adipokines and abdominal aortic calcification," Atherosclerosis, vol. 197, no. 1, pp. 428-434, 2008.

[8] M. Oikawa, T. Owada, H. Yamauchi et al., "Epicardial adipose tissue reflects the presence of coronary artery disease: comparison with abdominal visceral adipose tissue," BioMed Research International, vol. 2015, Article ID 483982, 7 pages, 2015.

[9] M. J. Pencina, R. B. D’Agostino Sr., R. B. D’Agostino Jr. et al., "Evaluating the added predictive ability of a new marker: from area under the ROC curve to reclassification and beyond," Statistics in Medicine, vol. 27, no. 2, pp. 157-172, 2008.

[10] G. A. Rosito, J. M. Massaro, U. Hoffmann et al., "Pericardial fat, visceral abdominal fat, cardiovascular disease risk factors, and vascular calcification in a community-based sample: the Framingham Heart study," Circulation, vol. 117, no. 5, pp. 605613, 2008.

[11] J. D. Miller, Y. Chu, R. M. Brooks, W. E. Richenbacher, R. Peña-Silva, and D. D. Heistad, "Dysregulation of antioxidant mechanisms contributes to increased oxidative stress in calcific aortic valvular stenosis in humans," Journal of the American College of Cardiology, vol. 52, no. 10, pp. 843-850, 2008.

[12] R. M. Weiss, M. Ohashi, J. D. Miller, S. G. Young, and D. D. Heistad, "Calcific aortic valve stenosis in old hypercholesterolemic mice," Circulation, vol. 114, no. 19, pp. 2065-2069, 2006.

[13] J. J. Kaden, C.-E. Dempfle, R. Grobholz et al., "Interleukin1 beta promotes matrix metalloproteinase expression and cell proliferation in calcific aortic valve stenosis," Atherosclerosis, vol. 170, no. 2, pp. 205-211, 2003.

[14] S. Furukawa, T. Fujita, M. Shimabukuro et al., "Increased oxidative stress in obesity and its impact on metabolic syndrome," The Journal of Clinical Investigation, vol. 114, no. 12, pp. 1752-1761, 2004.

[15] B. Gustafson, S. Hedjazifar, S. Gogg, A. Hammarstedt, and U. Smith, "Insulin resistance and impaired adipogenesis," Trends in Endocrinology and Metabolism, vol. 26, no. 4, pp. 193-200, 2015.

[16] J.-P. Després, "Body fat distribution and risk of cardiovascular disease: an update," Circulation, vol. 126, no. 10, pp. 1301-1313, 2012.

[17] T. Mazurek, L. Zhang, A. Zalewski et al., "Human epicardial adipose tissue is a source of inflammatory mediators," Circulation, vol. 108, no. 20, pp. 2460-2466, 2003.

[18] Y. Hirata, M. Tabata, H. Kurobe et al., "Coronary atherosclerosis is associated with macrophage polarization in epicardial adipose tissue," Journal of the American College of Cardiology, vol. 58, no. 3, pp. 248-255, 2011. 


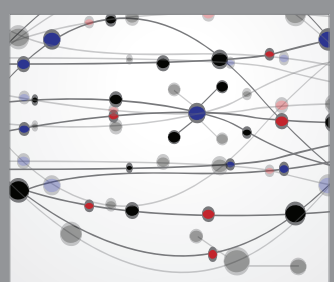

The Scientific World Journal
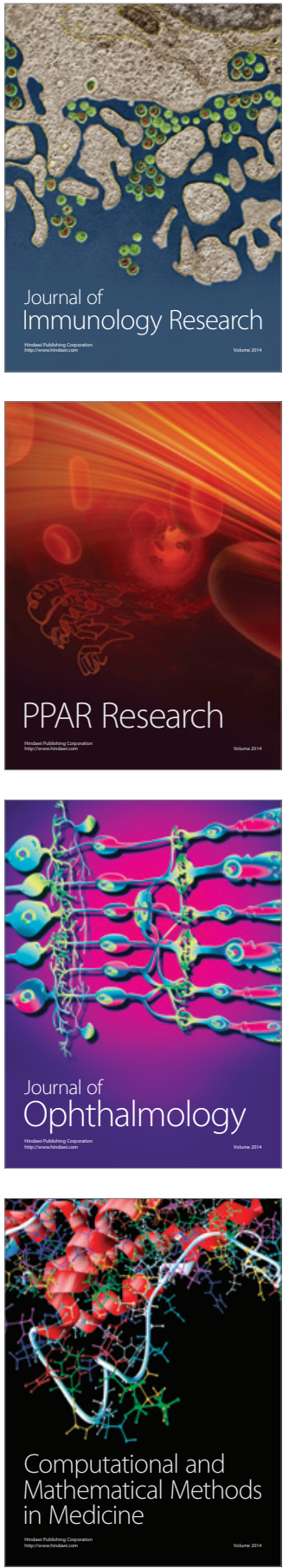

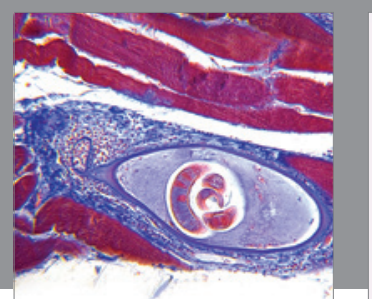

Gastroenterology Research and Practice

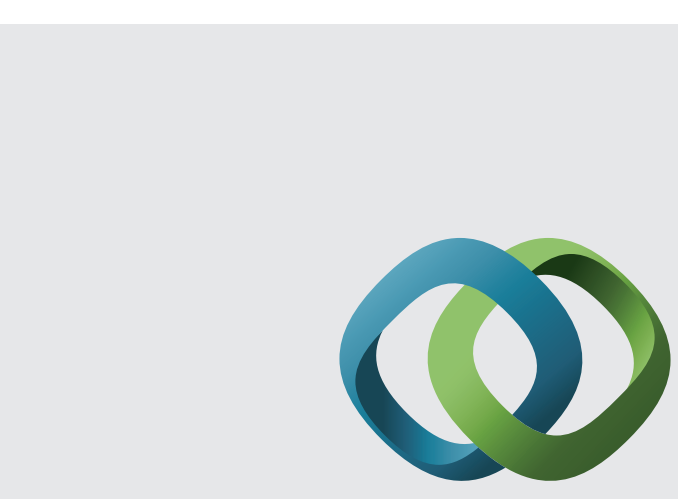

\section{Hindawi}

Submit your manuscripts at

http://www.hindawi.com
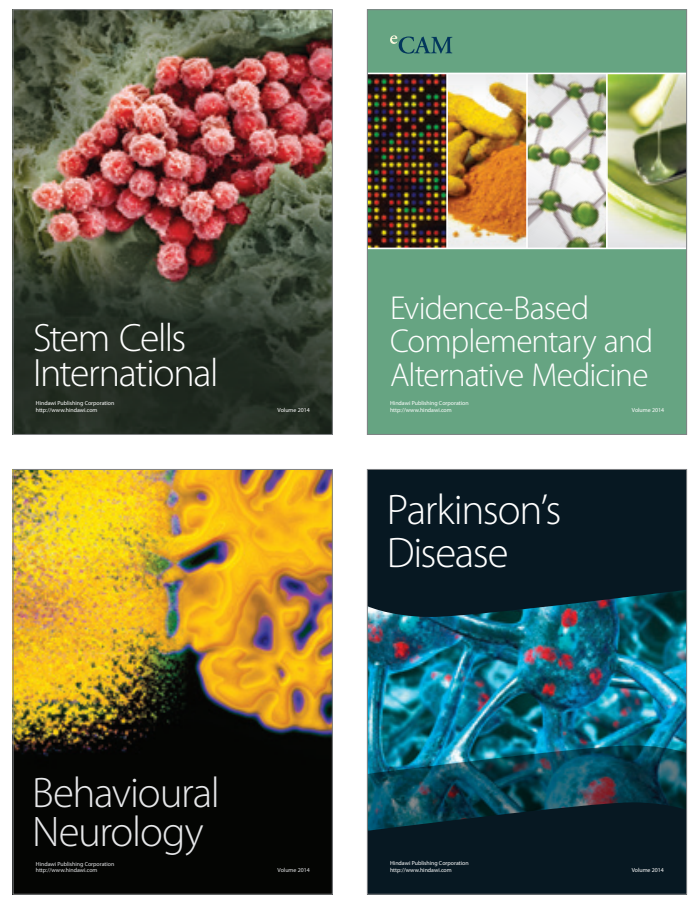
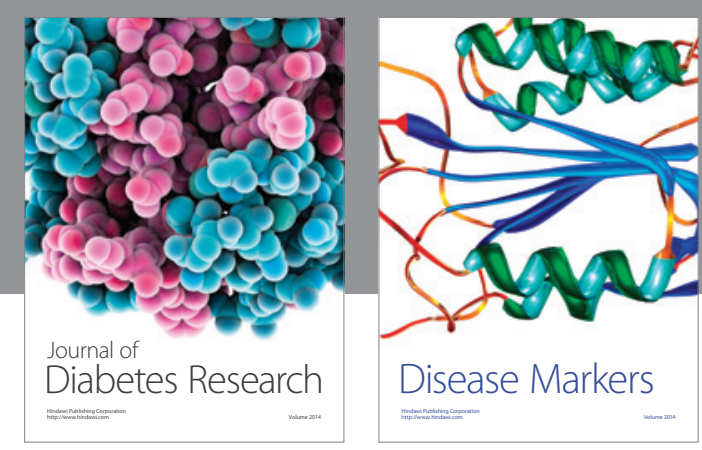

Disease Markers
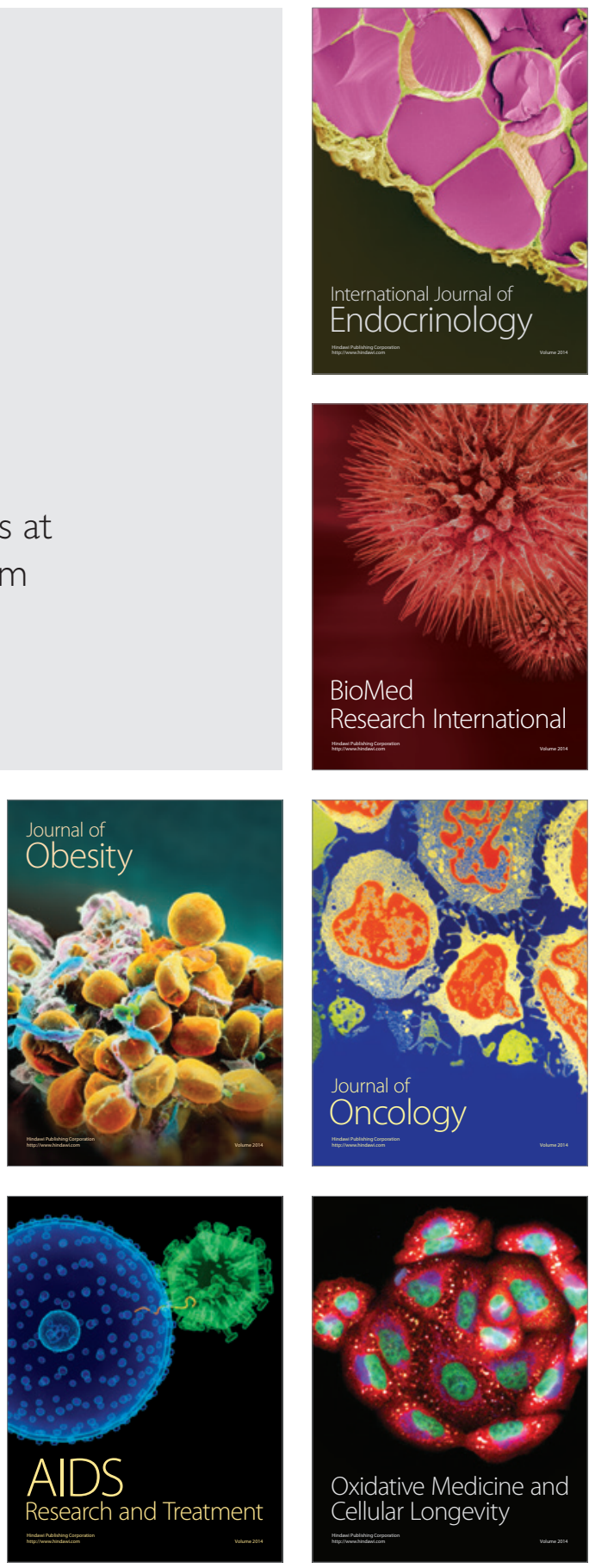